\title{
Structure Determination of Nickel-Embelin Complex
}

\author{
${ }^{1}$ Chepkwony S. C, ${ }^{2}$ Kiprono P. and ${ }^{3}$ Lutta S. \\ ${ }^{1,2,3}$ Department of Chemistry and Biochemistry, Faculty of Science, Chepkoilel University College, P.O. Box \\ 1125, Eldoret, Kenya
}

\begin{abstract}
Embelia schimperi is a plant belonging to the family of Myrsinaceae. The Myrsinaceae are chemotaxonomically associated with long alkyl side chain benzoquinones with embelin compound (1) being one of the benzoquinones. Isolation, constitution and synthesis of embelin (1) have already been effected but its capacity to form metallic complexes with all metals has not been studied so far. The chromatographic separation of an ethyl acetate extract from Embelia schimperi led to the isolation of a compound identified as 2,5-dihydroxy-3-methyl-1,4-benzoquinone (1) through spectroscopic analyses. Embelin (1) was modified by complexing it with nickel metal and found to form a complex under suitable conditions. The synthesis and structure determination of the metallic complex of embelin (1) have been described. The conductance measurement showed that the metallic complex formed was a non-electrolyte. The nickel metal is a good lewis acid since it has a great affinity for oxygen donors. Embelin (1) on the other hand, has electron donating carbonyl and hydroxyl oxygen atoms hence it reacts with the metal in an acid - base reaction. The Atomic Absorption Spectroscopy, Infrared Spectroscopy and ${ }^{1} H$ NMR Spectroscopy were used to determine the structure of the nickel - embelin complex.
\end{abstract}

Key Words: Embelia schimperi, Embelin, Nickel-embelin complex

\section{Introduction}

The Myrsinaceae or myrsine family is a rather large family from the order Ericales [1]. It consists of 35 genera and about 1000 species [2]. The transition metal - mediated reactions of organic compounds is of great importance since most metallic complexes have some therapeutic value or can be used as diagnostic agents [3]. Most metal chelates have also been found to have enhanced activities compared to the ligands [4]. Synthesis and characterization of zinc and copper complexes of embelin (1) that were purple and green in colour respectively have been successfully synthesized [5].

Nickel is an electropositive element of group 8 with an electron shell structure of $[\mathrm{Ar}] 3 \mathrm{~d}^{8} 4 \mathrm{~s}^{2}$. The most stable oxidation state of nickel is +2 , but compounds of $\mathrm{Ni}^{0}, \mathrm{Ni}^{+}$, and $\mathrm{Ni}^{3+}$ are well known, and $\mathrm{Ni}^{4+}$ has been demonstrated [6]. Complexes of nickel (II) are known in each of the common geometries; octahedral, tetrahedral and square planar (Housecroft and Sharpe, 2001).The square planar complexes are diamagnetic while the tetrahedral complexes are paramagnetic and a large ligand has to be used to force the tetrahedral configuration [7].

Reactions starting from $\mathrm{NiCl}_{2} \cdot 6 \mathrm{H}_{2} \mathrm{O}$ are used to form a variety of nickel coordination complexes because the $\mathrm{H}_{2} \mathrm{O}$ ligands are rapidly and easily displaced. In some derivatives, the chloride remains within the coordination sphere, whereas the chloride is displaced with highly basic ligands [8]. The aim of the research was to determine the structure formed by reaction of a nickel compound with embelin (1).

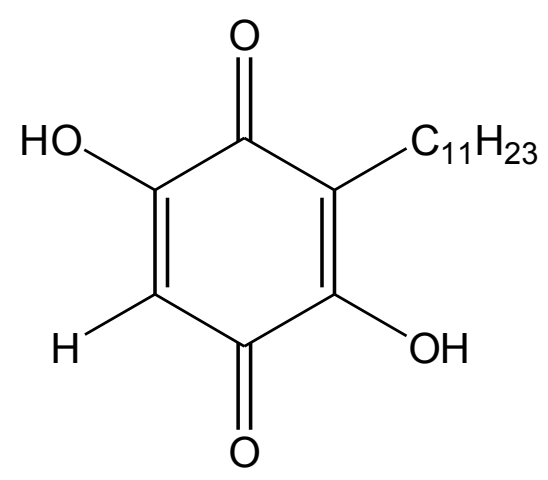

Embelin molecule (1) 


\section{Collection of plant parts}

\section{Materials And Methods}

Embelia schimperi stem barks were collected from mau forest in Kericho district which is about 260 $\mathrm{km}$ west of Nairobi city. Its identification was carried out at the herbarium, Department of Biological sciences, Chepkoilel University College and given a voucher number Ker 0205001 . The barks were air-dried for three weeks then ground into fine powder.

Extraction and isolation of embelin compound

A quantity of $1.2 \mathrm{~kg}$ of the bark powder of Embelia Schimperi was soaked in two litres of ethyl acetate in the cold for 48 hours while mechanically stirring. The extract was then filtered under suction and concentrated to dryness using a rotary evaporator to give a dark-brown sticky paste weighing 5.5 grams. This was then dissolved in n-hexane, mixed with 13.75 grams of de-activated silica gel which acted as the adsorbent and finally the solvent was removed from the mixture using a rotavapor. The slurry obtained was packed on a chromatographic column under n-hexane with 500 grams of de-activated silica gel. Elution was done using nhexane, ethyl acetate and methanol. The polarity of the system was increased with increased addition of ethyl acetate up to $100 \%$ ethyl acetate. The column was finally washed with methanol. Different fractions of the solvents were collected separately. Embelin (1) is highly unstable on non-deactivated silica gel. For this reason, the commercial analytical thin layer chromatography plates were deactivated by impregnating with $3 \%$ oxalic acid in methanol. This was done by dipping the plates for 2-3 minutes and then drying them for about 5 minutes in an oven at a temperature of $110^{\circ} \mathrm{C}$. The solvent system used to develop the thin layer chromatography plates was $n$-hexane: ethylacetate: acetic acid in the ratio of 85:10:5 respectively. The $n$-hexane fraction contained mainly fatty acid material. As the mobile phase became more polar by addition of ethyl acetate, the quinonoid pigments were eluted. This was confirmed by running a thin layer chromatography analysis. The compound that had an Rf of 0.525 , which was of interest, was in larger quantities than others and therefore masked the minor ones on thin layer chromatography plate. The compound, which was embelin (1), was recrystallized twice to obtain it in high purity. This was achieved by dissolving the 2.0 grams of the compound in ethyl acetate and leaving it to stand for one week. This yielded an orange compound which had a melting point of $140-142{ }^{\circ} \mathrm{C}$. This was in line with the melting point of embelin (1) given in literature [1].

Synthesis of nickel-embelin complex

Nickel (II) chloride, $\mathrm{NiCl}_{2} \cdot 6 \mathrm{H}_{2} 0,(0.0404 \mathrm{~g}, 0.17 \mathrm{mmols})$ was dissolved in $100 \mathrm{ml}$ methanol to give a green solution. Embelin (1), $\mathrm{C}_{17} \mathrm{H}_{26} \mathrm{O}_{4},(0.1000 \mathrm{~g}, 0.34 \mathrm{mmols})$ was dissolved in $150 \mathrm{ml}$ methanol to obtain an orange solution. The two solutions were then mixed, and the brown solution that resulted was stirred for two hours. The $\mathrm{pH}$ of the solution was measured at the end of the two hours. The solution was then concentrated to about $100 \mathrm{ml}$ by rotary evaporation, filtered and allowed to stand for 12 hours. The deep green product that precipitated was filtered and washed thoroughly with $150 \mathrm{ml}$ methanol, followed by $100 \mathrm{ml}$ acetone and finally $50 \mathrm{ml}$ diethyl ether. The resulting complex was air-dried for 24 hours, weighed and found to be 0.04 grams.

\section{Conductance measurement}

Conductivity measurements were done using an EC 215 conductivity meter at Chepkoilel University College. A standard solution of $0.01 \mathrm{M} \mathrm{KCl}$ was prepared and its conductance measured in order to determine the cell constant. The conductance of the nickel-embelin complex was then measured.

Structure determination of nickel-embelin complex

The complex was subjected to the following spectroscopic analyses: Atomic Absorption Spectroscopy using Chem Tech 2000 instrument at Moi University, Infrared spectroscopy using a Shimadzu model 408 spectrophotometer at Chepkoilel University College and Proton Nuclear Magnetic Resonance spectroscopy was recorded at $300 \mathrm{MHz}$ on an AV-300 NMR spectrometer at Surrey University.

\section{Results And Discussion}

Reaction of a solution of embelin (1) in methanol with methanol solution of nickel chloride crystals led to the formation $0.04 \mathrm{~g}$ of a deep green precipitate which upon dissolving with DMSO, formed a deep green solution. A characteristic feature of many d-block elements is the formation of coloured complexes which arise since the wavelength of light emitted or absorbed during d-d transition occurs in the visible region of the electromagnetic spectrum [9]. Conductance measurement was done in DMSO and the molar conductivity value of the nickel-embelin complex obtained was $0.19 \Omega^{-1} \mathrm{~cm}^{2} \mathrm{~mol}^{-1}$. This was below $50 \Omega^{-1} \mathrm{~cm}^{2} \mathrm{~mol}^{-1}$ which is the least value suggested by Greenwood and co-workers for electrolytes in DMSO [10]. This showed that the nickel-embelin complex formed was a non-electrolyte. 
The AAS study carried gave a $9.31 \%$ percentage composition of the nickel metal present in the complex. The theoretical percentage composition of nickel in the complex was found to be $8.62 \%$.

The IR spectrum of nickel-embelin complex showed that the carbonyl group shifted from $1610 \mathrm{~cm}^{-1}$ in embelin (1) to a broad peak in the region at $1530-1480 \mathrm{~cm}^{-1}$ with a maximum at $1520 \mathrm{~cm}^{-1}$ in the nickelembelin complex. While the hydroxyl group changed from a sharp peak at $3300 \mathrm{~cm}^{-1}$ in embelin (1) to a broad one in the region, $3400-3150 \mathrm{~cm}^{-1}$ in the nickel-embelin complex. The extreme case of protonation shows that the carbonyl bond has increased single-bond character and longer length, and the same tendency occurs in hydrogen bonding, leading to a lowering of the vibration frequency [11]. Studies done by Agarwal 2000, showed that a negative shift in carbonyl vibration indicates a decrease in the stretching force constant of carbonyl as a consequence of coordination through the carbonyl oxygen atom [12]. Thus, the carbonyl group in embelin (1) is coordinated to nickel through its oxygen atom. The change in hydroxyl group of embelin (1) is as a result of its coordination to nickel through its hydroxyl atom.

Table 1 shows some of selected IR bands of both embelin and the nickel-embelin complex.

Table 1. Some IR bands of embelin and nickel-embelin complex.

\begin{tabular}{|l|l|l|}
\hline \multicolumn{1}{|c|}{ IR BANDS $\left(\mathbf{c m}^{-1}\right)$} & EMBELIN & NICKEL-EMBELIN \\
\hline $\mathrm{O}-\mathrm{H}$ & 3300 & $3400-3150$ \\
\hline $\mathrm{C}-\mathrm{H}$ str. & 2900,2850 & 2900,2850 \\
\hline $\mathrm{C}=\mathrm{O}$ & 1610 & $1530-1480$ \\
\hline $\mathrm{C}-\mathrm{H}$ out of plane & 830,770 & 830,770 \\
\hline
\end{tabular}

The ${ }^{1} \mathrm{H}$ NMR studies of the nickel-embelin complex showed signals at $2.495 \mathrm{ppm}$ which was a peak due to the protons from the DMSO solvent used and a peak at $3.329 \mathrm{ppm}$ which was a peak caused by the terminal methyl group in the complex. Nickel being a $\mathrm{d}^{8}$ system has 2 unpaired electrons located in the $\mathrm{d}_{\mathrm{z} 2}$ and the $d_{x 2-y 2}$ orbitals. This causes it to form paramagnetic compounds. Hence, no signal is expected for NMR studies. This is due to the fact that the paramagnetic shift that occurs causes the ring carbons to broaden beyond recognition except those of the terminal methyl group. Hence, this affects the absorption of the carbon signals [4].

The structure of the nickel-embelin was determined to be as follows:

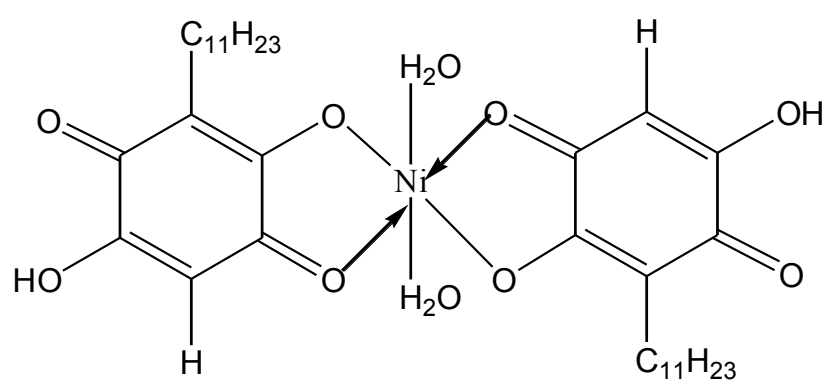

Nickel - embelin complex

Nickel metal contains empty d-orbitals that were utilized in bonding. Embelin (1) is expected to act as a bidentate ligand with possible coordination sites being the carbonyl and the hydroxyl functional groups. Embelin (1) contains an acidic proton which may be displaced by a base, and thus the nickel (II) ion behaves as a base and displaces the acidic proton from embelin (1). The nickel (II) ion which has a high affinity for oxygen and coordinates with ligands which contain oxygen as donor atoms is able to displace the acidic proton by accepting the lone pair of electrons from the hydroxyl oxygen atom of embelin (1), which possesses carbonyl and hydroxyl oxygen atoms. Thus, embelin (1) acts as an electron donor (lewis base) while nickel (II) acts as an electron acceptor (lewis acid).Therefore, the reaction between embelin (1) and nickel (II) ion is essentially an acid-base reaction.

\section{Conclusion}

The reaction of embelin with nickel ions leads to the formation of an octahedral complex. The two molecules of embelin provides four oxygen atoms around nickel and two water molecules from the nickel salt complete the coordination sphere to give an octahedral complex. 


\section{References}

[1]. Midiwo,J.O., Yenesew. A., Juma, B.F., Deres, S., Ayoo, J.A., Aluoch, A.O. and Gichu S. (2002). Bioactive compounds from some Kenyan Ethnomedicinal plants: Myrsinaceae, Polygonaceae and Psiadia punctulata. Phytochemistry. 311-323.

[2]. Manguro, L.O., Okwiri, S.O. and Lemmen,P. (2007). Oleanane-type triterpenes of Embelia schimperi leaves. Phytochemistry. 67 (24), 2641-50.

[3]. MacKay, K.M., MacKay, R.A. and Henderson, W. (2002). Introduction to Modern Inorganic Chemistry. $6^{\text {th }}$ Ed. 556-561

[4]. Kiremire, E., Massipa, S., Daniel, L. and Chibale, K. (2009). The synthesis, characterization and biological studies against malaria parasites of sulphur-based metal complexes of Cu (II), Cd (II), Co (II), Mn (II), Al (III) and Ag (I). (Unpublished work).

[5]. Cherutoi, J.K., Cheruiyot,L.L. and Kiprono, C.P. (2005). Synthesis and characterization of zinc (II) and copper (II) complexes of embelin. Bull.Chem.Soc.Ethiop. 19 (2), 295-299.

[6]. Housecroft, C.E. and Sharpe, A.G. (2001). Inorgnic Chemistry. Prentice Hall: Harlow.34.

[7]. Wikipedia, (2010). Crystal field theory. March 13.

[8]. Wikipedia, $(2010 \mathrm{c})$. Nickel chloride. July 2.

[9]. Henderson, W. (2002). Main group Chemistry. Royal Society of Chemistry. 161.

[10]. Greenwood, N.N., Straughan, B.P. and Wilson, A.E. (1968). J. Chem. Soc. A. 2209

[11]. Kemp, W. (1991). Organic Spectroscopy. $3^{\text {rd }}$ ed. Macmillan. London. 75.

[12]. Agarwal, R.K. and Agarwal, H. (2000). Some High Co-ordination Compounds of Lanthanides (III) derived from NIsonicotinamidosalicyaldimine. Bull. Chem. Soc. Ethiop. 14 (2), 143-153. 